\title{
Candida urinary tract infection and Candida species susceptibilities to antifungal agents
}

\author{
Kayo Osawa ${ }^{1}$, Katsumi Shigemura ${ }^{2}$, Hiroyuki Yoshida ${ }^{1}$, Masato Fujisawa ${ }^{2}$ and Soichi Arakawa ${ }^{1,2}$
}

The purpose of this study is to review Candida isolation from urine of urinary tract infection (UTI) patients over the recent 3 years at the Kobe University Hospital. We recorded the type of strain, the department where the patient was treated such as the intensive care unit (ICU), and combined isolation of Candida with other microorganisms. We investigated Candida isolation and susceptibilities to antifungal agents and analyzed the risk factors for combined isolation with other microorganisms. The most frequently isolated Candida was Candida albicans, which showed good (100\%) susceptibilities to 5 -fluorocytosine (5-FC) and fluconazole (FLCZ) but not to voriconazole (VRCZ), followed by C. glabrata. ICU was the greatest source of Candida-positive samples, and the most relevant underlying diseases of ICU patients were pneumonia followed by renal failure and post liver transplantation status. Combined isolation with other bacteria was seen in 27 cases (42.9\%) in 2009, 25 (33.3\%) in 2010 and $31(31.3 \%)$ in 2011 and comparatively often seen in non-ICU patients. Other candidas than C. albicans showed significantly decreased susceptibility to FLCZ over these 3 years $(P=0.004)$. One hundred $(97.1 \%)$ of 103 ICU cases were given antibiotics at the time of Candida isolation, and the most often used antibiotics were cefazolin or meropenem. In conclusion, $C$. albicans was representatively isolated in Candida UTI and showed good susceptibilities to 5-FC, FLCZ and VRCZ, but other candidas than $C$. albicans showed significantly decreased susceptibility to FLCZ in the change of these 3 years. The Journal of Antibiotics (2013) 66, 651-654; doi:10.1038/ja.2013.68; published online 26 June 2013

Keywords: Candida isolation; susceptibilities to antifungal agents; urinary tract infection

\section{INTRODUCTION}

The Infectious Diseases Society of America (IDSA) guideline was established in 2009 and it needs to evaluate the current trends of Candida urinary tract infection (UTI). ${ }^{1-3}$ Several researchers have stated that Candida infections basically emerge only in immunecompromised hosts with dosing such as steroids, immune suppressants or chemotherapy. ${ }^{2,4,5}$ However, other authors have found that while immune-compromised status is a risk factor for Candida infection, the patients with normal immune states may have a Candida infection, and culture tests should include those for fungus. ${ }^{6-9}$ In the absence of colonization, disseminated candidiasis is unlikely regardless of the presence of predisposing factors. ${ }^{7}$

Urinary tract mycosis may often result from Candida UTI because of Candida's frequency of isolation in urine. ${ }^{1,10,11}$ In general, Candida albicans is most often isolated in urine followed by C. glabrata or C. tropicallis. These strains show different trends in pathogenicity ${ }^{10,12,13}$ and combined isolation with other microorganisms. ${ }^{12}$ The detection of potential risk factors for Candida isolation are of clinical importance for understanding their extent of severities and for consideration of treatments, and Candida colonization may be considered as the factors for Candida infection. ${ }^{14-16}$
Regarding treatments for Candida, several established antifungal agents are commercially available. ${ }^{11,17-19}$ These drugs are classified based on their targeting points. ${ }^{20,21}$ Candida susceptibilities to several antifungal agents may be decreasing according to recent trends. ${ }^{22,23}$

In this study, we reviewed the recent cases of Candida isolation from urine from UTI patients and Candida susceptibilities to antifungal agents over the last 3 years at the Kobe University Hospital, Kobe, Japan.

\section{RESULTS}

\section{Candida isolation}

Urinary tract Candida spp. was isolated over the last 3 years as shown in Table 1. C. albicans was the most often isolated strain in all 3 years, followed by C. glabrata. Candida spp. was the most often isolated from intensive care unit (ICU) patients followed by the urological and general surgery departments (Table 1). Details of patients' backgrounds of ICU cases and their antibiotic uses before Candida isolation were shown in Tables 2 and 3. Shortly, the most relevant underlying diseases of ICU patients were pneumonia followed by renal failure or post-liver transplantation status. One hundred (97.1\%) out of 103 ICU cases were given antibiotics at the time of Candida isolation, and the median duration of antibiotics usage

\footnotetext{
${ }^{1}$ Infectious control team, Kobe University Hospital, Kobe, Japan and ${ }^{2}$ Division of Urology, Department of Organs Therapeutics, Faculty of Medicine, Kobe University Graduate School of Medicine, Kobe, Japan

Correspondence: Dr K Shigemura, Division of Urology, Department of Organs Therapeutics, Faculty of Medicine, Kobe University Graduate School of Medicine, 7-5-1 Kusunokicho, Chuo-ku, Kobe 650-0017, Japan.

E-mail: yutoshunta@hotmail.co.jp

Received 7 April 2013; revised 20 May 2013; accepted 29 May 2013; published online 26 June 2013
} 
Table 1 Patients' backgrounds

\begin{tabular}{lccc}
\hline & 2009 & 2010 & 2011 \\
\cline { 2 - 4 } $\mathrm{n}$ & 63 & 75 & 99 \\
\hline Inpatients department & $59(93.7 \%)$ & $70(93.3 \%)$ & $89(89.9 \%)$ \\
ICU & 34 & 28 & 41 \\
Urology & 7 & 5 & 5 \\
General surgeons & 3 & 4 & 6 \\
Gynecology & 2 & 6 & 4 \\
Cardiovascular & 0 & 5 & 3 \\
Digestive diseases & 1 & 5 & 2 \\
Others & 12 & 17 & 28 \\
Outpatients & $4(6.3 \%)$ & $5(6.7 \%)$ & $10(10.1 \%)$ \\
\hline
\end{tabular}

Abbreviation: ICU, intensive care unit.

Table 2 Details of ICU patients' backgrounds

\begin{tabular}{lr}
\hline $\mathrm{n}$ & 103 \\
\hline Gender & \\
Male & 42 \\
Female & 61 \\
& \\
Underlying diseases & \\
Pneumonia & 30 \\
Renal failure & 5 \\
Post liver transplantation status & 4 \\
Aortic aneurysm & 4 \\
Cerebral hemorrhage & 4 \\
Heart failure & 4 \\
Burns & 3 \\
Others & 49
\end{tabular}

Table 3 Antibiotics use before Candida isolation of ICU patients

\begin{tabular}{lc}
\hline Frequently used antibiotics & $\mathrm{n}$ \\
\hline Cefazolin & 35 \\
Meropenem & 35 \\
Vancomycin & 13 \\
Doripenem & 11 \\
Pazufloxacin & 11 \\
Sulbactam/ampicillin & 11 \\
Tazobactam/piperacillin & 10 \\
Cefozopran & 9 \\
Clindamycin & 6 \\
Imipenem & 5 \\
Cefpirome & 8 \\
Minocycline & 4 \\
Others & 25 \\
& \\
Antibiotic duration (days) & \\
Use & $100(97.1 \%)$ \\
$1 \sim 5$ & 30 \\
$6 \sim 10$ & 23 \\
$11 \sim 20$ & 31 \\
$21 \sim$ & 16 \\
Non-use & $3(2.9 \%)$ \\
\hline &
\end{tabular}

Table 4 Combined isolation of Candida spp. with other microorganisms

\begin{tabular}{|c|c|c|c|}
\hline & 2009 & 2010 & 2011 \\
\hline$n$ & 63 & 75 & 99 \\
\hline Single culture & 36 (57.1\%) & $50(66.7 \%)$ & $68(68.7 \%)$ \\
\hline ICU & $24(66.7 \%)$ & 23 (46.0\%) & 37 (54.4\%) \\
\hline Non-ICU & 12 (33.3\%) & 27 (54.0\%) & $31(45.6 \%)$ \\
\hline \multicolumn{4}{|l|}{ Organism } \\
\hline C. albicans & $21(58.3 \%)$ & 28 (56.0\%) & 35 (51.5\%) \\
\hline C. glabrata & 7 (19.4\%) & $11(22.0 \%)$ & 25 (36.8\%) \\
\hline C. parapsilosis & 5 (13.9\%) & $8(16.0 \%)$ & 2 (2.9\%) \\
\hline C. tropicalis & $3(8.3 \%)$ & $3(6.0 \%)$ & $4(5.9 \%)$ \\
\hline C. guilliermondii & 0 & 0 & $2(2.9 \%)$ \\
\hline Multiple cultures & $27(42.9 \%)$ & 25 (33.3\%) & 31 (31.3\%) \\
\hline ICU & $10(37.0 \%)$ & $5(20.0 \%)$ & $4(12.9 \%)$ \\
\hline Non-ICU & $17(63.0 \%)$ & 20 (80.0\%) & $27(87.1 \%)$ \\
\hline \multicolumn{4}{|l|}{ Kinds } \\
\hline Two kinds & 17 (63.0\%) & 18 (72.0\%) & $20(64.5 \%)$ \\
\hline Three kinds & $9(33.3 \%)$ & $5(20.0 \%)$ & $8(25.8 \%)$ \\
\hline Four kinds & $1(3.7 \%)$ & $1(4.0 \%)$ & $2(4.9 \%)$ \\
\hline$>$ Five kinds & 0 & $1(4.0 \%)$ & $1(2.4 \%)$ \\
\hline \multicolumn{4}{|l|}{ Organism } \\
\hline C. albicans & 21 (29.4\%) & 15 (45.5\%) & $24(58.5 \%)$ \\
\hline C. glabrata & 6 (17.6\%) & 12 (36.4\%) & $14(34.1 \%)$ \\
\hline C. parapsilosis & $1(2.9 \%)$ & $3(9.1 \%)$ & $2(4.9 \%)$ \\
\hline C. tropicalis & $6(17.6 \%)$ & $3(9.1 \%)$ & $1(2.4 \%)$ \\
\hline C. guilliermondii & 0 & 0 & 0 \\
\hline
\end{tabular}

Abbreviation: ICU, intensive care unit.

Table 5 Antifungal susceptibility (\%) of Candida spp

\begin{tabular}{lcccccc}
\hline Organism/antifungal & 2009 & 2010 & 2011 & $r$ & $p$ & $b$ \\
\hline C. albicans & & & & & & \\
$\quad$ Tested number of isolates & 37 & 35 & 52 & & & \\
5-FCa & 100 & 100 & 100 & - & - & - \\
FLCZ & 100 & 100 & 96.2 & 0.866 & 0.333 & -0.395 \\
ITCZ & 40.5 & 31.4 & 48.1 & 0.454 & 0.700 & 0.054 \\
VRCZ & 100 & 100 & 100 & - & - & - \\
& & & & & & \\
Non C. albicans a & & & & & & \\
Tested number of isolates & 21 & 33 & 46 & & & \\
5-FC & 100 & 100 & 100 & - & - & - \\
FLCZ & 47.6 & 33.3 & 15.2 & 0.998 & 0.004 & -0.061 \\
ITCZ & 100 & 100 & 100 & - & - & - \\
VRCZ & 90.5 & 87.9 & 89.1 & 0.538 & 0.638 & -0.413 \\
\hline
\end{tabular}

Abbreviations: 5-FC, 5-fluorocytosine; FLCZ, fluconazole; ITCZ, itraconazole; VRCZ,

voriconazole.

$r$, correlation coefficient and $b$, regression coefficient.

${ }^{a} \mathrm{C}$. glabrata, C. parapsilosis and C. tropicali.

was 10 days before Candida isolation. The median number of used antibiotics was two antibiotics and the most often used antibiotics were cefazolin and meropenem followed by vancomycin.

Our investigation of combined isolation with other microorganisms showed that 27 cases (42.9\%) of isolated Candida in 2009, 25 $(33.3 \%)$ in 2010 and 31 (31.3\%) in 2011 had combined isolation with 
other microorganisms (Table 4). Non-ICU patients tended more likely to have combined isolation (two or more kinds of Candida or combined isolation with other microorganisms) (63.0\% in 2009, $80.0 \%$ in 2010 and $87.1 \%$ in 2011). C. albicans was also the most frequently isolated in this context as well $(61.8 \%$ in $2009,45.5 \%$ in 2010 and $58.5 \%$ in 2011) followed by C. glabrata (17.6\% in 2009, $36.4 \%$ in 2010 and $34.1 \%$ in 2011). The microorganisms most commonly isolated in combination with Candidas were Enterococcus faecium, Pseudomonas aeruginosa or Enterococcus faecalis.

\section{Susceptibilities to antifungal agents}

We analyzed the data on Candida susceptibilities to representative antifungal agents. The data showed that over these 3 years C. albicans had good susceptibilities to 5-fluorocytosine (5-FC), fluconazole (FLCZ) and voriconazole (VRCZ); the susceptibility ratio was comparatively good (96.2 to $100 \%$ ) (Table 5). Other Candidas (C. glabrata, C. parapsilosis and C. tropicalis) had good susceptibilities to 5-FC and itraconazole (ITCZ) (100\%), whereas susceptibility to FLCZ have become significantly lower $(P=0.004)$ in the change of these 3 years investigated.

\section{DISCUSSION}

UTIs have been the focus of numerous studies, including optimal antibiotic treatments, the utility of urinary tract drainage, changes in causative microorganisms over time and in different regions and UTI categorization, such as complicated or uncomplicated UTI. ${ }^{11,24}$ This study focused on urinary tract mycosis, particularly Candida, which is comparatively often isolated from urine. ${ }^{1,11,25}$ We found that C. albicans and C. glabrata dominated our isolation of Candida from urine, representing about $90 \%$ of all the Candida cultured from our urine samples, and this high prevalence ratio is similar to that in other studies. ${ }^{10,12,13}$

Many cases involve multiple isolations with other microorganisms and Candida-related infections are also associated with immunecompromised patients. ${ }^{1,11}$ Immune-compromised patients easily become infected with mixed pathogenic microorganisms. ${ }^{1}$ Fisher et al. ${ }^{26}$ reported that C. albicans, compared with other Candida spp. such as C. glabrata, have the potential for strong adhesion, and this may be one reason for mixed infection or isolation with other microorganisms. The risks for these mixed isolations were reported by Sobel et al., ${ }^{12}$ who found that more than one species of Candida were found simultaneously in over $10 \%$ of patients, and candiduria frequently coexists with or follows bacteriuria.

Our data showed that mixed isolation (two or more kinds of Candida isolated or combined isolation with other microorganisms) tended to be seen more often in non-ICU patients. In general in our medical system, ICU patients are in a hyper-acute phase or status, so the duration of hospitalization or medical interventions in most cases is shorter than for non-ICU patients, a category that includes the cases with long-hospital admission and associated hospital exposure. In addition, our data showed pneumonia, renal failure and post liver transplantation status were often seen in ICU cases and most of them (97.1\%) were given antibiotics before Candida isolation. Fischer et al. ${ }^{27}$ revealed that the important risk factors for severe fungal infection were frequent or long-term medical exposure or treatments such as antibiotic dosing. Lundstrom and Sobel ${ }^{11}$ thought it is likely that antibiotics contribute to colonization by Candida spp. by suppressing endogenous bacterial flora. These works support our results and these kinds of studies need to be followed up in our medical system; debate persists on whether the Candida was isolated from UTI or colonization. Some authors have concluded that
Candida isolation from urine is mostly due to medical devices such as urinary tract catheters and should be regarded as colonization in most cases, ${ }^{25,28,29}$ requiring no treatments. Others conclude that Candida colonization is a definitive risk factor for Candida infections and these are often severe in immune-compromised hosts, including those undergoing chemotherapy or other immunesuppressant treatments, and in such cases Candida needs to be treated. ${ }^{1,2,11}$ We suggest, considering our results of severity especially in ICU-patients shown in Table 2, that the treatments in immunecompromised cases may be necessary like the latter opinions.

As to the susceptibilities to antifungal agents, our data showed that all our tested Candida spp. (C. albicans, C. glabrata, C. parapsilosis and C. tropicalis) had good susceptibilities to 5-FC, but other Candida than C. albicans showed decreased susceptibilities to FLCZ. These results were similar to those of Schmalreck et al. ${ }^{23}$ who also reported decreased susceptibilities to FLCZ and VRCZ in C. glabrata and C. tropicalis, respectively. They also reported that a decreased susceptibility to 5-FC was found in C. tropicalis. Pemán et al. ${ }^{30}$ showed that Candida spp. were all susceptible to 5-FC and VRCZ, but C. glabrata and C. krusei had decreased susceptibilities to these anti-fungal agents. Further, FLCZ failures of the treatment of $C$. glabrata causing renal infection have been reported with increasing frequency. ${ }^{31}$

We would like to emphasize the limitations of this study. First, this study is retrospective and includes all the urine materials isolated regardless of infection or colonization. Next, the number of Candida cases may not be enough for definitive conclusions, however, these are the consecutive cases of our institution. Third, the patients' backgrounds were investigated only from ICU patients partly because those of non-ICU patients had wide range of variation and were considered difficult to be analyzed. These limitations should be resolved in our future investigation of urinary tract Candida.

In conclusion, we documented urinary tract Candida isolation and combined isolation with other microorganisms and found that the latter tended to be seen more commonly in non-ICU patients. Other Candidas than C. albicans showed decreasing susceptibility to FLCZ in the change of these 3 years (2009-2011). Further approaches to address Candida infection will be based on this baseline study evaluating urinary tract Candida institutionally.

\section{METHODS}

\section{Patients}

Fungus was isolated from urine specimens from patients with UTI patients from January 2009 to December 2011 in Kobe University Hospital. We investigated the susceptibilities of isolated fungi to representative antifungal agents. UTI was defined as a symptomatic fungus isolation of $10^{5}$ or more colony forming units per $\mathrm{ml}$ in urine. We recorded the patient's sex, type of strain, departments treating the patient, combined isolation with other microorganisms and analyzed the patients especially focusing on the categorization of ICU or non-ICU patients and antibiotics usage before Candida isolation in ICU patients.

\section{Susceptibility testing}

Antifungal agents' susceptibilities were tested and the results were interpreted and reported using the reference broth microdilution method as described by the Clinical and Laboratory Standards Institute (CLSI) M27-S3 (2008. CLSI Document M100-S20). The MIC was defined as the lowest antimicrobial concentration that totally inhibited bacterial growth. Susceptibilities were evaluated by CLSI category. We tested Candida strains against the following antifungal agents by using drug susceptibility tests kit for yeast-like fungus (ASTY) (Kyokuto Pharmaceutical Industrial Co, Ltd, Takahagi, Japan) after $48 \mathrm{~h}$ of cultures: 5-FC, FLCZ, ITCZ and VRCZ. For quality control, C. parapsilosis ATCC22019 or C. Krusei ATCC6258 were used. 


\section{Statistical analyses}

Statistical analysis was conducted using linear regression analysis with PASW Statistics 17.0 software packages (for Windows; SPSS Inc., Chicago, IL, USA). The Candida susceptibilities of isolated strains to antifungal agents treatments were analyzed with linear regression analysis. Statistical significance was established at the 0.05 level.

1 Pappas, P. G. et al. Clinical Practice Guidelines for the Management of Candidiasis: 2009 Update by the Infectious Diseases Society of America. Clin. Infect. Dis 48, 503-535 (2009).

2 Bukhary, Z. A. Candiduria: a review of clinical significance and management. Saudi. J. Kidney. Dis. Transpl. 19, 350-360 (2008).

3 Fisher, J. F., Sobel, J. D., Kauffman, C. A. \& Newman, C. A. Candida urinary tract infections-treatment. Clin. Infect. Dis. 52, S457-S466 (2011).

4 Wise, G. J. \& Silver, D. A. Fungal infections of the genitourinary system. J. Urol. 149, 1377-1388 (1993).

5 Wainstein, M. A., Graham, R. C. Jr \& Resnick, M. I. Predisposing factors of systemic fungal infections of the genitourinary tract. J. Urol. 154, 160-163 (1995).

6 Reagan, D. R., Pfaller, M. A., Hollis, R. J. \& Wenzel, R. P. Characterization of the sequence of colonization and nosocomial candidemia using DNA fingerprinting and a DNA probe. J. Clin. Microbiol. 28, 2733-2738 (1990).

7 Pittet, D. et al. Contour-clamped homogeneous electric field gel electrophoresis as a powerful epidemiologic tool in yeast infections. Am. J. Med. 91, S256-S263 (1991).

8 Voss, A., Hollis, R. J., Pfaller, M. A., Wenzel, R. P. \& Doebbeling, B. N. Investigation of the sequence of colonization and candidemia in nonneutropenic patients. J. Clin Microbiol. 32, 975-980 (1994).

9 Toya, S. P., Schraufnagel, D. E. \& Tzelepis, G. E. Candiduria in intensive care units: association with heavy colonization and candidaemia. J. Hosp. Infect 66, 201-206 (2007).

10 Kauffman, C. A. et al. Prospective multicenter surveillance study of funguria in hospitalized patients. The National Institute for Allergy and Infectious Diseases (NIAID) Mycoses Study Group. Clin. Infect. Dis. 30, 14-18 (2000).

11 Lundstrom, T. \& Sobel, J. Nosocomial candiduria: a review. Clin Infect Dis. 32, 1602-1607 (2001).

12 Sobel, J. D. et al. Candiduria: a randomized, double-blind study of treatment with fluconazole and placebo. The National Institute of Allergy and Infectious Diseases (NIAID) Mycoses Study Group. Clin. Infect. Dis 30, 19-24 (2000).

13 Guler, S., Ural, O., Findik, D. \& Arslan, U. Risk factors for nosocomial candiduria. Saudi Med. J. 27, 1706-1710 (2006).

14 Kumar, M. S., Cridge, P., Molavi, A., Stephan, R. \& Abouna, G. M. Infectious complications in the first 100 days after renal transplantation. Transplant. Proc. 27, 2705-2706 (1995).
15 Whiting, P. et al. Clinical effectiveness and cost-effectiveness of tests for the diagnosis and investigation of urinary tract infection in children: a systematic review and economic model. Health. Technol. Assess. 10, 1-154 (2006).

16 Rivera-Sanchez, R. et al. Prospective study of urinary tract infection surveillance after kidney transplantation. BMC. Infect. Dis. 10, 245 (2010).

17 Jacobs, L. G. Fungal urinary tract infections in the elderly: treatment guidelines. Drugs Aging. 8, 89-96 (1996).

18 Fisher, J. F. Candiduria: when and how to treat it. Curr. Infect. Dis. Rep. 2, 523-530 (2000).

19 Pappas, P. G. et al. Guidelines for treatment of candidiasis. Clin. Infect. Dis. 38, 161-189 (2004)

20 Perea, S. \& Patterson, T. F. Antifungal resistance in pathogenic fungi. Clin. Infect. Dis. 35, 1073-1080 (2002)

21 Rajeshkumar, R. \& Sundararaman, M. Emergence of Candida spp. and exploration of natural bioactive molecules for anticandidal therapy-status quo. Mycoses, 55, e60-73 (2012).

22 Pfaller, M. A et al. Results from the ARTEMIS DISK Global Antifungal Surveillance Study, 1997 to 2007: a 10.5-year analysis of susceptibilities of Candida Species to fluconazole and voriconazole as determined by CLSI standardized disk diffusion. J. Clin. Microbiol. 48, 1366-1377 (2010).

23 Schmalreck, A. F. et al. Species and susceptibility distribution of 1062 clinical yeast isolates to azoles, echinocandins, flucytosine and amphotericin B from a multi-centre study. Mycoses 55, e124-137 (2012).

24 Arakawa, S. et al. Usefulness and problems of the urinary tract infection criteria for evaluating drug efficacy for complicated urinary tract infections. J. Infect. Chemother 13, 279-284 (2007).

25 Platt, R., Polk, B. F., Murdock, B. \& Rosner, B. Risk factors for nosocomial urinary tract infection. Am. J. Epidemiol. 124, 977-985 (1986).

26 Fisher, J. F., Kavanagh, K., Sobel, J. D., Kauffman, C. A. \& Newman, C. A. Candida urinary tract infection: pathogenesis. Clin. Infect. Dis. 52, S437-S451 (2011).

27 Fisher, J. F. et al. Urinary tract infections due to Candida albicans. Rev. Infect. Dis. 4, 1107-1118 (1982).

28 Berrouane, Y. F., Herwaldt, L. A. \& Pfaller, M. A. Trends in antifungal use and epidemiology of nosocomial yeast infections in a university hospital. J. Clin. Microbiol. 37, 531-537 (1999).

29 Shay, A. C. \& Miller, L. G. An estimate of the incidence of Candiduria among hospitalized patients in the United States. Infect. Control. Hosp. Epidemiol. 25, 894-895 (2004).

30 Pemán, J. et al. Epidemiology, species distribution and in vitro antifungal susceptibility of fungaemia in a Spanish multicentre prospective survey. J. Antimicrob. Chemother 67, 1181-1187 (2012)

31 Ansari, S. H., Levin, M. H. \& Lipshitz, S. Re: Fluconazole treatment in Torulopsis glabrata upper urinary tract infection causing ureteral obstruction. J Urol. 154, 1870 (1995) 